\title{
BMJ Open Skin and wound care for individuals with graft versus host disease: a scoping review protocol
}

\author{
Jill Campbell (10 , ${ }^{1,2}$ Nicole Gavin, ${ }^{2,3}$ Elise Button, ${ }^{2,3}$ Natasha Roberts ${ }^{3,4}$
}

To cite: Campbell J, Gavin N, Button $\mathrm{E}$, et al. Skin and wound care for individuals with graft versus host disease: a scoping review protocol. BMJ Open 2020;10:e038567. doi:10.1136/ bmjopen-2020-038567

- Prepublication history for this paper is available online. To view these files, please visit the journal online (http://dx.doi org/10.1136/bmjopen-2020038567).

Received 16 March 2020 Revised 28 July 2020 Accepted 28 August 2020

\section{Check for updates}

\section{Author(s) (or their} employer(s)) 2020. Re-use permitted under CC BY-NC. No commercial re-use. See rights and permissions. Published by BMJ.

${ }^{1}$ Skin Integrity Service, Royal Brisbane and Women's Hospital, Herston, Queensland, Australia ${ }^{2}$ School of Nursing, Queensland University of Technology, Brisbane, Queensland, Australia ${ }^{3}$ Cancer Care Services, Royal Brisbane and Women's Hospital, Herston, Queensland, Australia

${ }^{4}$ School of Public Health and

Social Work, Queensland

University of Technology,

Brisbane, Queensland, Australia

Correspondence to

Dr Jill Campbell;

jill.campbell@qut.edu.au

\section{ABSTRACT}

Introduction Graft versus host disease (GVHD) is a major cause of morbidity and mortality following allogenic haematopoietic stem cell transplantation. It is an immunological reaction, involving many organs, leading to a wide range of clinical manifestations. Cutaneous manifestations are the most common sign of GVHD, as well as pain, vulnerability to infection and impaired quality of life.

Despite the burdens that cutaneous GVHD presents for patients, their carers and the healthcare system, limited evidence is available to guide day to day supportive skin care and wound management. Our objective is to conduct a scoping review to map the evidence for skin and wound management and identify evidence-practice gaps for individuals with acute or chronic cutaneous GVHD.

Methods and analysis Our review will follow the scoping review methodological framework developed by Arksey and O'Malley and further refined by the Joanna Briggs Institute Scoping Review Methods Manual. Databases to be searched include; PubMed, EMBASE, PsycINF0, Cochrane Central Register of Controlled Trials, Cumulative Index to Nursing and Allied Health Literature, Web of Science and MEDLINE from 1970 to February 2020. Database searches will be supplemented with searches from relevant reference lists and grey literature. Descriptive statistical analyses will be performed

Ethics and dissemination This scoping review does not require ethical approval. Findings will be disseminated through a peer-reviewed publication and conference presentation.

\section{INTRODUCTION}

\section{Background}

Graft versus host disease (GVHD) is a major cause of morbidity and mortality following allogenic haematopoietic stem cell transplantation. ${ }^{1}$ It is an immunological reaction, involving many organs most notably skin and gut. It can affect between $40 \%$ and $60 \%$ of patients and accounts for $15 \%$ mortality following transplantation. ${ }^{2}$ There are two main types of GVHD, acute and chronic, which differ in pathogenesis, time of onset and clinical presentation. Acute GVHD usually has a limited course, but can be life threatening, while chronic GVHD can require

\section{Strengths and limitations of this study}

This study will use a rigorous approach to scoping reviews to map supportive skin care and wound management of individuals with graft versus host disease and identify evidence gaps for this population.

- The review will encompass literature not previously synthesised.

- The scoping review will include systematic reviews, randomised, non-randomised and observational studies.

- Grey literature and documents from relevant organisations will be included.

long term treatment with immunosuppressive therapies which in turn place patients at risk of major complications specifically risk of infection. ${ }^{3}$ Cutaneous manifestations are the most common sign of GVHD. Individuals suffering from cutaneous GVHD experience a variety of manifestations, including erythematous maculopapular morbilliform eruptions, follicular erythema, erythematous macular and popular rashes erythroderma, itching, dysthesia, sclerotic changes, pain, vulnerability to infection and impact on wellbeing and quality of life. ${ }^{124}$ There is evidence available to guide skin-directed treatments such as topical or systemic medications and specialist therapies such as phototherapy and extracorporeal photopheresis. ${ }^{4-6}$ However, a significant evidence gap regarding day to day supportive skin care (including cleansing and moisturising, keeping skin healthy and intact, providing comfort and supporting wellbeing), ${ }^{7}$ and wound management procedures (including dressing and product selection, treatment regimens, pain, itch and other symptom management), remains.

Maintaining healthy intact skin is imperative for patients with cutaneous GVHD, however, providing even routine skin hygiene can present enormous challenges. Clinical decision making regarding supportive skin 
care and wound management for this cohort is complex, often requiring multidimensional interventions. Due to immunosuppressive therapies, the risk of infection is an ongoing threat for these patients, ${ }^{56}$ and must be a primary consideration in skin and wound management plans. In addition, prevention and management of xeroses, pain, skin injury and further deterioration of skin barrier function are critical factors in skin and wound management for these patients. Complex clinical decisions include skin and wound assessment, development of individualised evidence-based care plans which include skin hygiene regimes, formulation of appropriate wound management plans, appropriate product selection including emollients, skin cleansers, wound cleansing products, wound dressings, antimicrobial agents, adhesive and dressing retention products, prevention and management of skin or wound pain and/or itch. While there may be pathophysiological and presentation differences between acute and chronic cutaneous GVHD, the principles of supportive skin care and wound treatment are primarily determined by patient presentation, symptoms and preferences.

Anecdotally, many wound dressings are poorly tolerated by these patients, as they are frequently uncomfortable, exacerbate pain and itch, and often cause extreme distress during application and particularly removal. Medical adhesive products commonly used for dressing retention are unsuitable as they can cause skin trauma, and extreme pain on removal, making retention of wound dressings challenging. Furthermore, dressing procedures can take considerable time, require significant analgesia, and are physically and psychologically burdensome for the patient and the care provider.

Despite the common physical, psychological and economic burden the management of cutaneous GVHD presents for patients, their carers and the healthcare system, very little evidence is available to guide the complex and targeted supportive skin and wound management required. Skin and wound management for this group can be ad hoc, and may be reliant on clinician preference, cost or even product procurement practices, rather than guided by evidence. We believe that a scoping review may contribute to the development of evidence-based skin and wound management guidelines for patients with acute and chronic cutaneous GVHD.

A preliminary search for existing scoping reviews on the topic has been conducted. Databases searched included PubMed, EMBASE and Medline.

\section{Rationale}

Literature examining the medical treatment of acute and chronic cutaneous GVHD is extensive, however, there is a paucity of evidence to inform supportive skin care and wound management. To further advance the field of supportive skin care and wound management for these patients, gaps in the literature need to be identified. This scoping review will identify evidence gaps and provide the foundation for future research to address the evidencepractice gap that exists for these patients.

\section{Objectives}

The principal objective of this scoping review is to map the existing literature on supportive skin care and wound management for patients with acute or chronic GVHD. Specifically, our objectives are to:

- Conduct a systematic search of peer-reviewed and grey literature to scope the available evidence regarding supportive skin care and wound management for adults with acute or chronic cutaneous GVHD.

- Identify practice gaps regarding the supportive skin care and wound management of adult patients with acute or chronic cutaneous GVHD.

\section{METHODS}

This scoping review protocol was developed using the methodological framework proposed by Arksey and $\mathrm{O}^{\prime}$ Malley ${ }^{8}$ and further refined by the Joanna Briggs Institute. ${ }^{9}$ The approach describes five methodological stages: (1) identifying the research questions, (2) identifying relevant studies, (3) selecting studies, (4) charting the data, (5) collating, summarising and reporting the results. The scoping review protocol follows the Preferred Reporting Items for Systematic Reviews and Meta-Analyses, Scoping Review (PRISMA-ScR) checklist. ${ }^{10}$

\section{Stage 1: identifying the research questions}

To construct the research questions, we used the mnemonic population, concept and context (PCC). ${ }^{9}$ In this study, the population is adults with acute or chronic cutaneous GVHD, the concept is supportive skin care and wound management, and the context is any healthcare setting. We have identified two research question to guide the scoping review; what evidence is available regarding supportive skin care and wound management in adults with cutaneous acute or chronic cutaneous GVHD in any healthcare setting, and what are the practice gaps regarding the supportive skin care and wound management of adult patients with acute or chronic cutaneous GVHD? Secondary research questions are: (1) what types of research on cutaneous GVHD has been conducted? (2) What population demographics and clinical settings were included? (3) What were the interventions, interventional themes studied? (4) What outcome measures were reported?

\section{Stage 2: identifying relevant studies Eligibility criteria}

Inclusion will meet the following criteria; empirical and theoretical studies of any study design (eg, randomised controlled trials, cohort, case-control, quasiexperimental, cross-sectional and qualitative studies) quantitative research, case reports, literature reviews, scope reviews guidelines, policy, protocols, theses and dissertations published in the study data bases, and published in English. Studies must refer to supportive skin 
care or wound management for adults aged 18 years or older suffering from acute or chronic cutaneous GVHD.

Studies will be excluded if they investigate exclusively skin-directed treatments including drug interventions (either local or systemic), phototherapy or extracorporeal photopheresis for acute or chronic cutaneous GVHD or in any way other than defined herewith.

A systematic search strategy will be employed to identify the relevant studies for inclusion in the review. The following electronic databases have been selected: (1) PubMed; (2) EMBASE, PsycINFO; (3) Cochrane Central Register of Controlled Trials, Cumulative Index to Nursing and Allied Health Literature (CINAHL), Web of Science and MEDLINE. Bone marrow transplants commenced in the early 1970s, ${ }^{11}$ therefore the search period will be from January 1970 until December 2020.

A scan of grey literature will be conducted. ${ }^{12}$ Websites of relevant organisations and agencies will be scanned for any documents related to supportive skin and wound management for cutaneous GVHD in adults. Organisations will be identified by recommendations from the research team and from a preliminary search of the internet via Google. Examples of such organisations include American Society of Clinical Oncology (ASCO), American Society of Haematology, Oncology Nursing Society, Haematology Society of Australia and New Zealand (HSANZ), Cancer Nurses Society of Australia (CNSA), European Society for Blood and Marrow Transplantation, European Haematology Association, British Society for Haematology (BSH). Reference lists will be hand searched for further studies.

\section{Search strategy}

The search (figure 1) was guided by Boolean operators 'AND', and 'OR' as necessary. A three-step search strategy will be used consistent with Joanna Briggs Institute (JBI) guidelines. ${ }^{9}$ An initial limited search was conducted in the online databases MEDLINE and CINAHL, followed by an analysis of text words in the title and abstracts and of

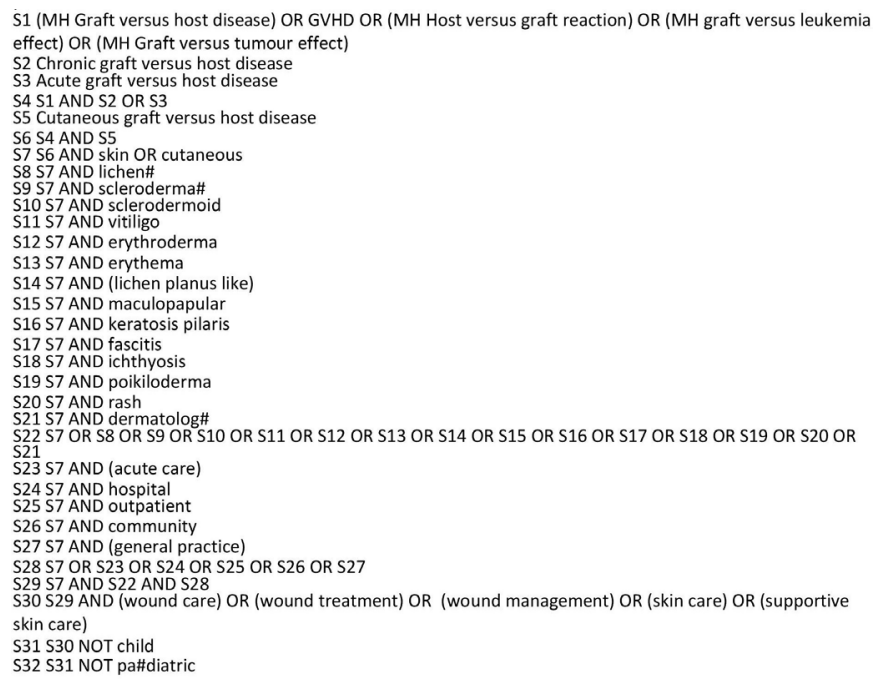

Figure 1 Search strategy. the index terms used to describe the articles. See figure 1 for the search strategy for MEDLINE. This informed the development of a search strategy, that will be adapted for each database or information source. The reference list of all articles included will be screened for additional sources. A specialist nursing and health librarian from Queensland University of Technology assisted with the development of the search strategy.

\section{Stage 3: study selection}

The review process will consist of two levels of screening: (1) title and abstract review and (2) a full text review. For the initial screening, two investigators will independently review titles and abstracts of articles retrieved in the search to identify potentially eligible articles. The retrieved titles will be exported into the reference manager EndNote (Clarivate Analytics), which will subsequently be used to remove the duplicates. The number of included and excluded studies at this level will be recorded together with documentation of the screening decisions. These decisions will be based on screening of the title and abstract and include: (1) studies both reviewers agree to include will move to the second level of the screening process, (2) studies that both reviewers agree to exclude will not be read in full and excluded from the review and (3) studies where there is non-agreement regarding exclusion, or where title and abstract screening is inconclusive, the study will be included in the second round for full text review before a decision is made.

In the second level of the screening stage, two investigators will independently assess the full text articles to determine whether they meet the eligibility criteria. Disagreement will be resolved with by discussion with a third investigator until consensus is reached. All inclusion and exclusion decisions will be documented, Cohen's kappa coefficient will be calculated as a measure of interrater reliability. ${ }^{13}$

\section{Stage 4: charting the data}

A specific data extraction tool will be developed for this review by two reviewers to determine which variables to extract. The tool will be tested by two independent reviewers before use to ensure all relevant results are extracted. Relevant data will include author, year of publication, origin/country of origin, study design, or type of paper, aims/purpose, population, sample size, methods, intervention type (supportive skin care or wound management), comparator and duration of intervention (if applicable), outcomes and outcome measures (if applicable), analgesia, adverse effects and key findings that relate to the scoping review objectives. ${ }^{9}$ Studies will be randomly assigned to each reviewer and data will be charted by two reviewers independently, and verified by two other reviewers. The data extraction tool will be adapted as necessary as an iterative process during the review, with the final version included in the scoping review report. If further information is required, we will contact authors for clarification. All decisions regarding changes to data 
extraction tool or to the protocol will be documented and reported. ${ }^{10}$

\section{Stage 5: collating, summarising and reporting the results}

All information regarding selection of sources will be presented in a flow diagram according to the PRISMA extension for reviews (PRISMA-ScR): checklist and explanation. ${ }^{10}$ The studies will be grouped by the type of intervention, that is supportive skin care and wound management. Frequency counts will be will be used to report included study characteristics and interventions. ${ }^{14}$ These results will be reported in the following formats (1) tabular summary including study design or article type and general study characteristics, (2) diagrammatic representation of the results to map the evidence and (3) a narrative synthesis of the evidence and results. Anticipated heterogeneity of the studies means that data or pooling and meta-analysis will not be conducted.

We expect there may be a limited number of prospective clinical trials, retrospective studies or other literature that address the objectives of this scoping review. Should this be the case, the authors intend to publish these results, as identifying the gap in evidence guiding supportive skin care and wound management for this group will constitute an important finding of the review. Implications for research and practice will be reported.

\section{Patient and public involvement}

Patients and public were not involved in the development of this scoping review protocol. Results or the completed review will be disseminated to patients and public by presenting the findings via organisations such as Leukaemia Foundation Australia, Leukaemia and Lymphoma Society and Leukaemia UK.

\section{DISCUSSION AND CONCLUSION}

The aim of this review is to identify and classify evidence to improve the care of patients with skin and wound management needs as a result of cutaneous GVHD. We believe the understanding of the breadth of evidence available in this field may contribute to development of care protocols that will improve outcomes for these patients. This scoping review is part of a larger project that as a longerterm goal aims to understand the skin and wound issues faced by patients who develop cutaneous GVHD following allogenic haematopoietic stem cell transplantation and to develop interventions and protocols to improve care. This review could be an important advance in the care of skin and wound problems in those with GVHD.

Skin and wound problems are common in patients with cutaneous GVHD, and impact on quality of life, and disease burden for these patients. ${ }^{136}$ However, there remains a gap in the evidence available to guide skin and wound care for this group. Therefore, an urgent imperative exists to address this gap. This review will make a substantial contribution understanding the available evidence and may contribute to improved care and subsequent outcomes for these patients.

\section{ETHICS AND DISSEMINATION}

Scoping review methodology consists of reviewing data from publicly available material, ethical approval is not required. The results of this review will be disseminated to stakeholders such as consumers, policy makers, clinicians and professional organisations, for example, ASCO, HSANZ, CNSA and BSH, via conference presentations and educational events such as workshops and webinars.

Acknowledgements The authors thank Sarah Howard, Liaison Librarian (Health), at Queensland University of Technology (QUT) for her guidance and consultation in the development of the systematic database search strategy for this protocol.

Contributors All authors contributed to the preparation, drafting and editing of this scoping review protocol. JC conceived the idea and developed the research questions, developed the research plan and conducted the initial review. JC, NG, EB and NR developed the data extraction tool and the systematic database search strategy in consultation with the specialist health librarian at QUT. All authors contributed to the preparation and editing of the manuscript, and approved the final version of this manuscript.

Funding The authors have not declared a specific grant for this research from any funding agency in the public, commercial or not-for-profit sectors.

Competing interests None declared.

Patient and public involvement Patients and/or the public were not involved in the design, or conduct, or reporting, or dissemination plans of this research.

Patient consent for publication Not required.

Provenance and peer review Not commissioned; externally peer reviewed.

Open access This is an open access article distributed in accordance with the Creative Commons Attribution Non Commercial (CC BY-NC 4.0) license, which permits others to distribute, remix, adapt, build upon this work non-commercially, and license their derivative works on different terms, provided the original work is properly cited, appropriate credit is given, any changes made indicated, and the use is non-commercial. See: http://creativecommons.org/licenses/by-nc/4.0/.

ORCID iD

Jill Campbell http://orcid.org/0000-0002-1253-8831

\section{REFERENCES}

1 Lee SJ, Flowers MED. Recognizing and managing chronic graftversus-host disease. Hematology Am Soc Hematol Educ Program 2008:134-41.

2 Strong Rodrigues K, Oliveira-Ribeiro C, de Abreu Fiuza Gomes S, et al. Cutaneous graft-versus-host disease: diagnosis and treatment. Am J Clin Dermatol 2018;19:33-50.

3 Mawardi H, Hashmi SK, Elad S, et al. Chronic graft-versus-host disease: current management paradigm and future perspectives. Oral Dis 2019;25:931-48.

4 Department of Clinical Haematology. Guidelines for diagnosis and management of cutaneous graft versus host disease Oxford university hospitals, 2017. Available: http://nssg.oxford-haematology. org.uk/bmt/gvhd/B-2-7c-diagnosis-and-management- cutaneousgvhd.pdf [Accessed 20 Nov 2019].

5 Kim YJ, Lee GH, Kwong BY, et al. Evidence-based, skin directed treatments for cutaneous chronic graft-versus-host disease. Cureus 2019;11:e6462.

6 Dignan FL, Clark A, Amrolia P, et al. Diagnosis and management of acute graft-versus-host disease. Br J Haematol 2012;158:30-45.

7 Kottner J, Surber C. Skin care in nursing: a critical discussion of nursing practice and research. Int J Nurs Stud 2016;61:20-8.

8 Arksey H, O'Malley L. Scoping studies: towards a methodological framework. Int J Soc Res Methodol 2005;8:19-32.

9 Peters M, Godfrey C, Mclnerney P, et al. The Joanna Briggs Institute reviewers' manual 2015: methodology for JBI scoping reviews, 2015. Available: http://joannabriggs.org/assets/docs/sumari/Reviewers- 
Manual_Methodology-for-JBI-Scoping- Reviews_2015_v2.pdf [Accessed 20 Nov 2019].

10 Tricco AC, Lillie E, Zarin W, et al. PRISMA extension for scoping reviews (PRISMA-ScR): checklist and explanation. Ann Intern Med 2018;169:467-73.

11 Ezzone S, Schmit-Pokorny K. Blood and marrow stem cell transplantation; principles practice and nursing insights. 3 edn. Sudbury, Mass: Jones and Bartlett Publishers, 2007.
12 Godin K, Stapleton J, Kirkpatrick SI, et al. Applying systematic review search methods to the grey literature: a case study examining guidelines for school-based breakfast programs in Canada. Syst Rev 2015;4:138

13 Cohen J. Weighted kappa: nominal scale agreement with provision for scaled disagreement or partial credit. Psychol Bull 1968;70:213-20.

14 Levac D, Colquhoun H, O'Brien KK. Scoping studies: advancing the methodology. Implement Sci 2010;5:69. 